\title{
Sub-Micrometer-Sized Spectrometer by Using Plasmonic Tapered Channel-Waveguide
}

\author{
Da Eun Lee, Tae-Woo Lee, and Soon-Hong Kwon* \\ Department of Physics, Chung-Ang University, Seoul 156-756, Korea
}

(Received September 29, 2014 : revised October 7, 2014 : accepted October 13, 2014)

\begin{abstract}
It has been a critical issue to reduce the size of spectrometers in many fields such as on-chip chemical and biological sensing. The proposed plasmonic channel-waveguide with a sub-micrometer width has a cutoff frequency which enables us to control wavelength dependent propagation properties. We focused on the capability of the waveguide for spectral-to-spatial mapping when the waveguide width changes gradually. In this paper, we propose a plasmonic tapered channel-waveguide structure as a compact spectrometer with a physical size of $0.24 \times 2.0 \times 0.20 \mu^{3}$. The scattering point just above the tapered waveguide moves linearly depending on the wavelength of the injecting light. The spectral-to-spatial mapping can be improved by increasing the tapered length.
\end{abstract}

Keywords : Nanophotonics, Plasmonics, Compact spectrometer

OCIS codes : (240.6680) Surface plasmons; (230.0230) Optical devices; (130.7408) Wavelength filtering devices

\section{INTRODUCTION}

Optical spectrometers have a long history from early human civilization to today. Their key characteristic of spectral-to-spatial mapping is widely applicable in chemical and biological fields. Recently, it has become very important to reduce size and integrate devices in order to construct a lab-on-a-chip device. However, the primitive grating-based-spectrometers should have large enough size to support diffraction and imaging of light, thus they have an intrinsic limit to miniaturizing their operating size [1-4]. Many studies of the spectrometer with micrometer scale [5-9] have come out recently, while the sub-micrometer region is still a challenge to design and operate.

Plasmonic channel-waveguide, which can be designed as a sub-micrometer cavity to apply to a nanolaser $[10,11]$ or a refractive index sensor [12], has been studied in recent years. The dispersion curve of the square channel-waveguide has a cutoff frequency, and adjacent two channel-waveguides with different widths make a mode gap since the cutoff frequency shifts depending on the waveguide width. Cutoff frequency increases by decreasing the width, thus the narrower waveguide acts as a mirror. The previous studies used this mechanism to build a Fabry-Perot cavity by placing two narrow waveguides to both the left and the right of a broader square channel-waveguide cavity. The structures achieved a compact photon confinement with only a few hundred nanometers size, including the cavity and side waveguides [11, 12].

In this study, we propose a plasmonic tapered channelwaveguide structure as a sub-micrometer-sized spectrometer. This spectrometer uses the cutoff mechanism to control a scattering point depending on the wavelength, which is able to perform spectral-to-spatial mapping by observing the movement of that point. The tapered channel-waveguide structure is an on-chip structure with a physical size of $0.24 \times 2.0 \times 0.20 \mu \mathrm{m}^{3}$. We used the finite-difference time-domain (FDTD) method to investigate the characteristics of the tapered spectrometer. We will discuss the scattering behaviors in the proposed structure for different incident wavelengths.

\section{BASIC CONCEPT}

\subsection{Plasmonic Square Channel-waveguide}

Our spectrometer is based on a plasmonic square channel-waveguide with width of $w=240 \mathrm{~nm}$ and depth of $d=200 \mathrm{~nm}$, filled by dielectric with refractive index of $n=3.4$, as shown in Fig. 1(a). The waveguide has simple structure with a square shaped groove on the metal substrate,

\footnotetext{
*Corresponding author: soonhong.kwon@gmail.com

Color versions of one or more of the figures in this paper are available online.
} 


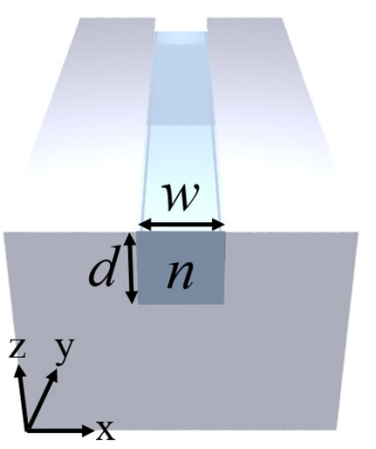

(a)

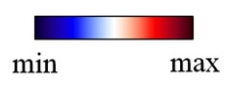

(b)

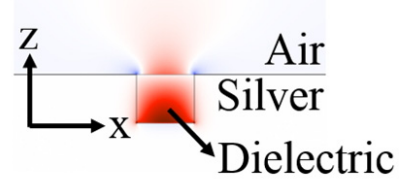

FIG. 1. (a) Schematic of a dielectric filled plasmonic square channel-waveguide structure with $w=240 \mathrm{~nm}, d=200 \mathrm{~nm}, n$ $=3.4$. (b) Electric field $\left(\mathrm{E}_{\mathrm{z}}\right)$ profile of the waveguide mode with wavelength $1776 \mathrm{~nm}$.

and covered by dielectric material at the bottom of the waveguide. We suppose the metal to be silver since it has low metallic absorption loss over the visible-infrared range in simulation. Also, a background material is assumed to be air. The surface plasmon-polariton wave (SPP wave) propagates through the waveguide, confined on the metaldielectric interface. Figure 1(b) is an electric field profile of the surface plasmon mode with a wavelength of 1776 $\mathrm{nm}$ in this channel-waveguide. The electric field is strongly confined at the bottom interface. In the calculation, silver is assumed to be a Drude metal, represented by the equation $\varepsilon(\omega)=\varepsilon_{\infty}-\omega_{p}^{2} /\left(\omega^{2}+i \gamma \omega\right)$, where Drude parameters are as follows: the background dielectric constant $\varepsilon_{\infty}=3.1$, the plasma frequency $\omega_{p}=1.4 \times 10^{16} \mathrm{~s}^{-1}$, and the collision frequency $\gamma=3.1 \times 10^{13} \mathrm{~s}^{-1}[10-13]$. The grid size of 20 $\mathrm{nm}$ is used in the simulations.

\subsection{Dispersion Property and Cutoff Frequency}

We investigated frequency dependencies of the channelwaveguide mode on the waveguide width. Figure 2(a) shows the dispersion relations of the channel-waveguide mode for different widths of $120 \mathrm{~nm}$ (black), $160 \mathrm{~nm}$ (red), and $200 \mathrm{~nm}$ (blue). The curves move to lower frequency region for larger waveguide width. This is because field cross-section increases when the waveguide width increases.

In this plasmonic square channel-waveguide, there exist cutoff frequencies at the wave vector of zero, indicated by red circles in Fig. 2(a). The cutoff frequency for the waveguide with $w=120 \mathrm{~nm}$ is $1404 \mathrm{THz}, w=160 \mathrm{~nm}$ is $1204 \mathrm{THz}$, and $w=200 \mathrm{~nm}$ is $1061 \mathrm{THz}$. In the frequency region lower than the cutoff frequency, the SPP wave cannot propagate along that waveguide because of absence of any mode. Our spectrometer uses this cutoff mechanism to prohibit propagation of the injected wave to the smaller width waveguide, as a narrow waveguide acts as a mirror.

Figure 2(b) is a plot of cutoff wavelength as a function of the waveguide width. At the waveguide width of 80

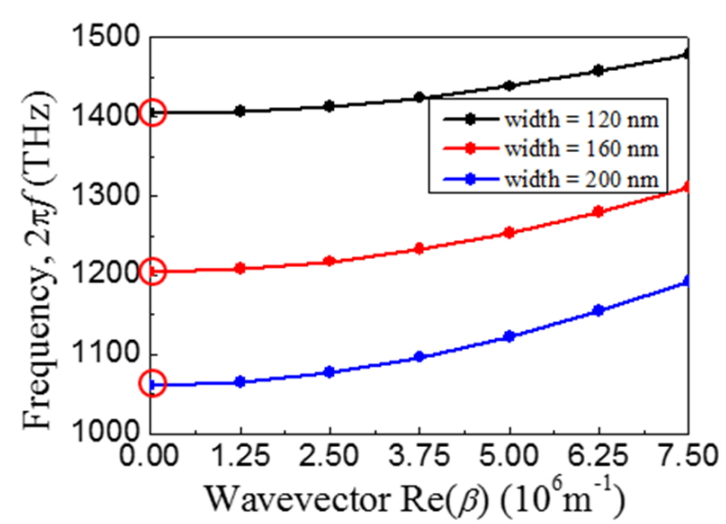

(a)

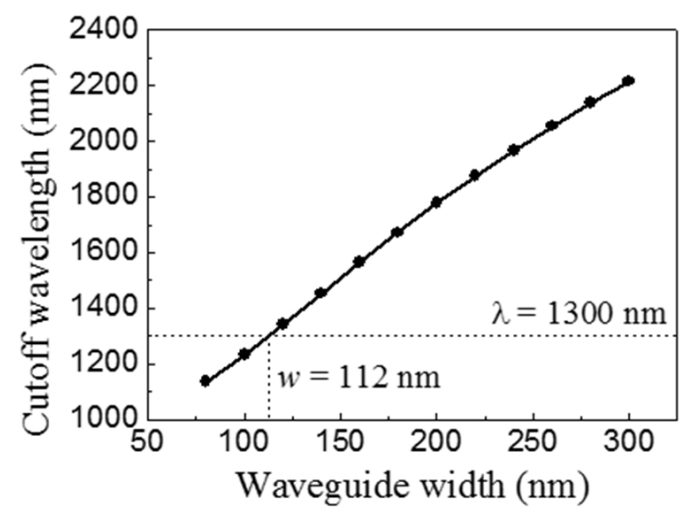

(b)

FIG. 2. (a) Dispersion curves and (b) cutoff wavelength dependency on the waveguide width $w$ of the channel-waveguide.

$\mathrm{nm}$, the cutoff wavelength is $1135 \mathrm{~nm}$, and at the width of $300 \mathrm{~nm}$, the cutoff wavelength becomes $2216 \mathrm{~nm}$. Cutoff wavelength linearly increases by increasing waveguide width. When the incident wavelength is assumed to be $1300 \mathrm{~nm}$, the cutoff width is estimated to be $112 \mathrm{~nm}$.

\section{RESULTS}

\subsection{Plasmonic Tapered Channel-waveguide}

We designed a tapered channel-waveguide consisting of two widths, an entrance width, $w_{a}=240 \mathrm{~nm}$ and an exit width, $w_{b}=40 \mathrm{~nm}$, tapered length $l=2000 \mathrm{~nm}$ and depth $d=200 \mathrm{~nm}$, filled with dielectric of refractive index $n=$ 3.4 (Fig. 3(a)). In the tapered region, as waveguide width decreases, the dispersion curve continuously moves to the upper frequency region, and the cutoff frequency increases as the wave propagates into the narrower region. When the cutoff wavelength at a certain point of the waveguide corresponding to wavelength of the incident source, the wave cannot proceed from the point anymore. Therefore, the injected light source keeps propagating through the channel-waveguide, while it is blocked if it reaches the cutoff point, and then the wave reflects. This results in 


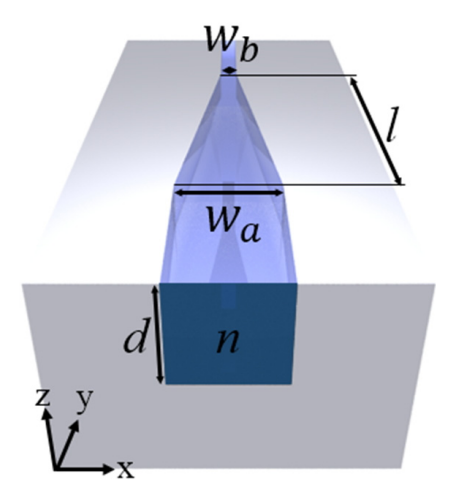

(a)

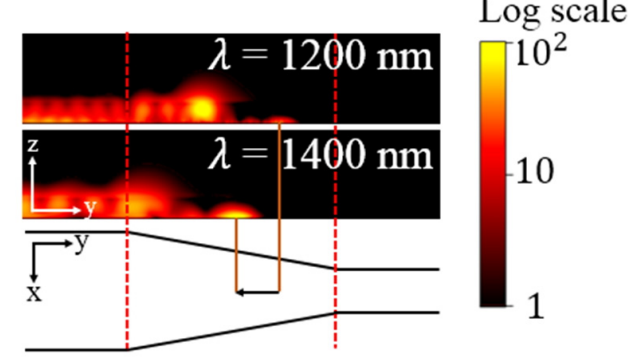

(c)

FIG. 3. (a) Schematic of a plasmonic tapered channel-waveguide with structure parameters, $w_{a}=240 \mathrm{~nm}, w_{b}=40 \mathrm{~nm}, d=200$ $\mathrm{nm}, l=2000 \mathrm{~nm}$. (b), (c) One period time averaged electric field intensity profiles $\left(E^{2}\right)$ in log scale at $x-y$ plane (b) and $y-z$ plane (c) for the $\lambda=1200 \mathrm{~nm}$ and $1400 \mathrm{~nm}$, respectively.

scattering as well as reflections near the cutoff point. This scattered wave enables us to measure the change of the reflection point, which we will discuss later.

Figure 3(b), (c) shows propagation and reflections at the tapered region for the SPP waves with wavelengths of $1200 \mathrm{~nm}$ and $1400 \mathrm{~nm}$ in the waveguide. Here, the time averaged $|E|^{2}$ field images are represented as log scale at the $x-y$ plane (b) and the $y-z$ plane (c). The tapered structure at right (b) and below (c) the electric field intensity images indicates how the reflection point changes depending on the source wavelength. The reflection points are $1460 \mathrm{~nm}$ for $\lambda=1200 \mathrm{~nm}$ and $1040 \mathrm{~nm}$ for $\lambda=1400 \mathrm{~nm}$ from the starting point of the tapered region. As shown in Fig. 3(b) and (c), the source with shorter wavelength propagates more than that with longer wavelength. This wavelength dependent SPP propagation can be applied to spatial mapping of the spectrum, which allows the tapered waveguide to act as a spectrometer.

\subsection{Detection of the Scattering Wave}

As a practical matter, it is hard to observe the movement of the reflection point at the bottom of the narrow waveguide. However, the open-cell structure of the proposed channelwaveguide allows the SPP wave to scatter at the reflection point, and the scattered wave moves depending on the reflection point. This scattered wave can easily be detected and mapped by the camera as an intensity distribution in the $x-y$ plane. For this reason, we investigated time averaged up-going Poynting vector $\left(\mathrm{S}_{\mathrm{z}}\right)$ images in the $\mathrm{x}-\mathrm{y}$ plane at height $h=500 \mathrm{~nm}$ from the top of the waveguide. Figure 4(a) shows that a bright, well-localized, single spot is observed at the Poynting vector image for the wavelength range from $1200 \mathrm{~nm}$ to $1400 \mathrm{~nm}$ with $50 \mathrm{~nm}$ steps. The bright spots, which would be observable by a camera, occur at $y$ $=860 \mathrm{~nm}$ for $\lambda=1200 \mathrm{~nm}$ and at $\mathrm{y}=300 \mathrm{~nm}$ for $\lambda=$ $1400 \mathrm{~nm}$. The spot moves to near the entrance, into the large width region, as the incident wavelength becomes longer. This result coincides with what we observed from the SPP wave. Here, we set the start point of the tapered region as $\mathrm{y}=0$, and the images are normalized by each of their max intensities in linear scale. In spite of light absorption and scattering of light, the short size of the proposed structure allows reasonable detected light power, which is approximately $10 \%$ of the input power. It would be sufficient to be used for a practical spectrometer. The output ratio can be further increased by putting in a grating or by changing the height of the waveguide.

Figure $4(\mathrm{~b})$ is a plot of the scattering position versus source wavelength from $1200 \mathrm{~nm}$ to $1400 \mathrm{~nm}$ for different tapered spectrometer with $l=1000 \mathrm{~nm}$ (black) and $l=$ $2000 \mathrm{~nm}$ (red). The x-crossed data are the expected reflection points by cutoff frequencies in dispersion curves from Fig. 2 , where the waveguide width corresponds to the point where the cutoff wavelength becomes equal to the incident wavelength. Scattering points are estimated from the vertical Poynting vector images in the tapered waveguide structure of Fig. 3(a) and the calculated reflection points are obtained from the dispersion curves of the simple channel waveguide in Fig. 1(a). The two lines with the same color, which represent expected reflection point (cross symbol) and scattering point (circle), show similar slopes for both cases, $l=1000 \mathrm{~nm}$ and $l=2000 \mathrm{~nm}$, respectively, except for the position differences. This position differences are mainly attributed to the fact that the imaged scattering spot does not occur exactly above the reflection point. As waveguide width decreases, the horizontal wave vector component along the waveguide decreases and the vertical wave vector component becomes large, resulting in scattering out into the free space. Therefore, the scattering spot is always observed before the reflection point where the horizontal wave vector component becomes zero. As the wavelength increases with $50 \mathrm{~nm}$ step, the scattering spot moves to narrower waveguide region and the movements are clearly observable. Therefore, the scattering image from the proposed tapered channel-waveguide provides the wavelength information of the incident light by spatial mapping.

In Fig. 4(b), the slope of the graph, which indicates spatial distribution of the different wavelengths, strongly depends on the tapered length $l$. For $l=1000 \mathrm{~nm}$, scattering position moves $\Delta y=220 \mathrm{~nm}$ by wavelength change of $\Delta \lambda$ $=200 \mathrm{~nm}$ from $1200 \mathrm{~nm}$ to $1400 \mathrm{~nm}$. However, for the case of $l=2000 \mathrm{~nm}$, the same wavelength change results 


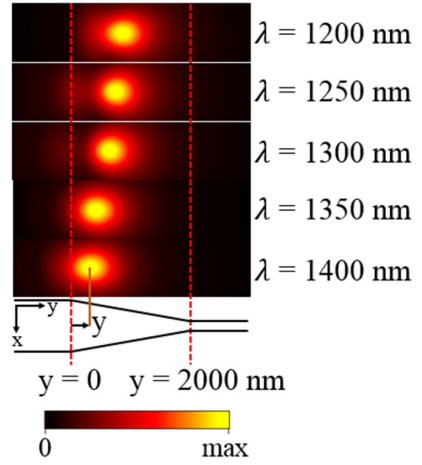

(a)

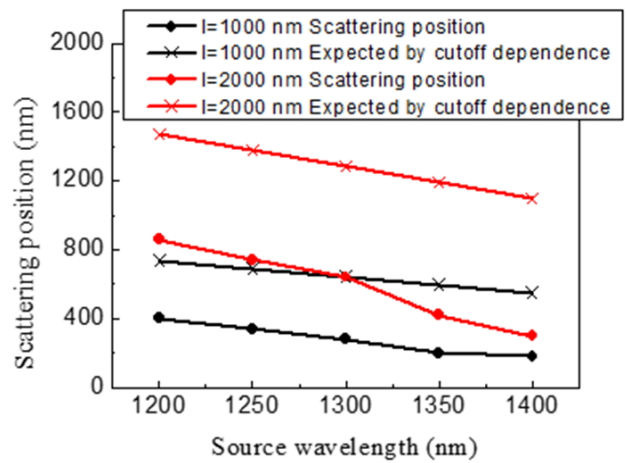

(b)

FIG. 4. (a) Time averaged outgoing Poynting vector $\left(\mathrm{S}_{\mathrm{z}}\right)$ images in $\mathrm{x}-\mathrm{y}$ plane at $h=500 \mathrm{~nm}$ from the top of the waveguide for different wavelengths from $1200 \mathrm{~nm}$ to 1400 $\mathrm{nm}$ by five steps with a step of $50 \mathrm{~nm}$. (b) Plot of the scattering position versus the wavelength of the light source by vertical Poynting vector image (circle symbol) and expectation (cross symbol). Black lines represent the data for tapered length of $l$ $=1000 \mathrm{~nm}$, and red lines are of $l=2000 \mathrm{~nm}$.

in the much larger shift of $\Delta y=560 \mathrm{~nm}$. Thus the slope increased by 2.55 times when this tapered length is doubled. This relation between the scattering position and the tapered length is due to the decrease of the reduction rate of the waveguide width as the tapered length increases. From the result, increase of the tapered length allows us to measure the source wavelength with a higher spectral resolution. The spectral resolution of the proposed structure can be obtained by the full-width half-maximum (FWHM) size of the scattering spot and the wavelength dependence of the spatial movement $(\Delta y=560 \mathrm{~nm}$ for the wavelength change, $\Delta \lambda=200 \mathrm{~nm}$ from $1200 \mathrm{~nm}$ to $1400 \mathrm{~nm}$, in the structure with $l=2000 \mathrm{~nm}$ ), which is approximately $105 \mathrm{~nm}$. The spectral resolution can be inversely proportional to the tapered length due to increased movement of the scattering position.

\section{CONCLUSION}

We proposed a plasmonic tapered square channel-waveguide as a sub-micrometer-sized spectrometer. The tapered channelwaveguide can map the spectrum image by spectral-to-spatial dependent scattering wave, based on cutoff wavelength mechanism. We investigated the dispersion curves of the channel-waveguide with several waveguide widths, and the cutoff wavelength dependences on the waveguide width, to analyze and expect the characteristics of this type of spectrometer. The cutoff wavelength shows linear dependency on the waveguide width. We applied the width dependence to design tapered channel-waveguide, and used the cutoff mechanism to control the scattering position which indicates the wavelength of the incident light. Also, in order to provide a practical measurement scheme, we calculated vertical Poynting vector images of the up-going scattering wave upper the waveguide top. The single, localized bright spot that occurred in the images moved linearly by change of the source wavelength. Therefore, since the spot position has spectral dependence, one can map the spectrum of the incident light to spatial intensity distribution. As a result of the calculation, the spot moved clearly for the wavelength difference of $50 \mathrm{~nm}$. In addition, for the tapered length of $2000 \mathrm{~nm}$, the wavelength change of $200 \mathrm{~nm}$ from $1200 \mathrm{~nm}$ to $1400 \mathrm{~nm}$ results in the large scattering spot movement of $\Delta y=560 \mathrm{~nm}$. In addition, our spectrometer takes on-chip structure with a physical size of $0.24 \times 2.0 \times 0.20 \mathrm{\mu m}^{3}$.

\section{ACKNOWLEDGMENT}

This research was supported by the Chung-Ang University Excellent Student Scholarship. This work was supported in part by the National Research Foundation of Korea through the Korean Government under Grant NRF 2013R1A2A2A 01014491.

\section{REFERENCES}

1. S. H. Kong, D. D. L. Wijngaards, and R. F. Wolffenbuttel, "Infrared micro-spectrometer based on a diffraction grating," Sens. Actuators A 92, 88-95 (2001).

2. S. Janz, A. Balakrishnan, S. Charbonneau, P. Cheben, M. Cloutier, A. Delage, K. Dossou, L. Erickson, M. Gao, P. A. Krug, B. Lamontagne, M. Packirisamy, M. Pearson, and D.-X. Xu, "Planar waveguide Echelle gratings in silica-onsilicon," IEEE Photonics 16, 503-505 (2004).

3. T. Fukazawa, F. Ohno, and T. Baba, "Very compact arrayedwaveguide-grating demultiplexer using $\mathrm{Si}$ photonic wire waveguides," Jpn. J. Appl. Phys. 43, L673-L675 (2004).

4. P. Cheben, J. H. Schmid, A. Delage, A. Densmore, S. Janz, B. Lamontagne, J. Lapointe, E. Post, P. Waldron, and D.-X. Xu, "A high-resolution silicon-on-insulator arrayed waveguide grating microspectrometer with sub-micrometer aperture waveguides," Opt. Express 15, 2299-2306 (2007).

5. B. Momeni, E. S. Hosseini, M. Askari, M. Soltani, and A. Adibi, "Integrated photonic crystal spectrometers for sensing 
applications," Opt. Commun. 282, 3168-3171 (2009).

6. B. B. C. Kyotoku, L. Chen, and M. Lipson, "Sub-nm resolution cavity enhanced micro-spectrometer," Opt. Express 18, 102-107 (2010).

7. Z. Xia, A. A. Eftekhar, M. Soltani, B. Momeni, Q. Li, M. Chamanzar, S. Yegnanarayanan, and A. Adibi, "High resolution on-chip spectroscopy based on miniaturized microdonut resonators," Opt. Express 19, 12356-12364 (2011).

8. H. Leong and J. Guo, "A surface plasmon resonance spectrometer using a super-period metal nanohole array," Opt. Express 20, 21318-21323 (2012).
9. B. Redding, S. F. Liew, R. Sarma, and H. Cao, "Compact spectrometer based on a disordered photonic chip," Nat. Photonics 7, 746-751 (2013).

10. M.-K. Seo, S.-H. Kwon, H.-S. Ee, and H.-G. Park, "Full three-dimensional subwavelength high-Q surface-plasmonpolariton cavity," Nano Lett. 9, 4078-4082 (2009).

11. J.-H. Kang, H.-G. Park, and S.-H. Kwon, "Room-temperature high-Q channel-waveguide surface plasmon nanocavity," Opt. Express 19, 13892-13898 (2011).

12. S.-H. Kwon, "Ultrasmall plasmonic cavity for chemical sensing," Plasmonic 8, 963 (2013).

13. P. B. Johnson and R. W. Christy, "Optical constants of the noble metals," Phys. Rev. B 6, 4370-4379 (1972). 